\title{
COVID-19 detection from chest $x$-ray using MobileNet and residual separable convolution block
}

\author{
V. Santhosh Kumar Tangudu ${ }^{1} \cdot$ Jagadeesh Kakarla $^{1} \cdot$ Isunuri Bala Venkateswarlu ${ }^{1}$
}

Accepted: 10 November 2021 / Published online: 28 January 2022

(c) The Author(s), under exclusive licence to Springer-Verlag GmbH Germany, part of Springer Nature 2021

\begin{abstract}
A newly emerged coronavirus disease affects the social and economical life of the world. This virus mainly infects the respiratory system and spreads with airborne communication. Several countries witness the serious consequences of the COVID-19 pandemic. Early detection of COVID-19 infection is the critical step to survive a patient from death. The chest radiography examination is the fast and cost-effective way for COVID-19 detection. Several researchers have been motivated to automate COVID-19 detection and diagnosis process using chest x-ray images. However, existing models employ deep networks and are suffering from high training time. This work presents transfer learning and residual separable convolution block for COVID-19 detection. The proposed model utilizes pre-trained MobileNet for binary image classification. The proposed residual separable convolution block has improved the performance of basic MobileNet. Two publicly available datasets COVID5K, and COVIDRD have considered for the evaluation of the proposed model. Our proposed model exhibits superior performance than existing state-of-art and pre-trained models with 99\% accuracy on both datasets. We have achieved similar performance on noisy datasets. Moreover, the proposed model outperforms existing pre-trained models with less training time and competitive performance than basic MobileNet. Further, our model is suitable for mobile applications as it uses fewer parameters and lesser training time
\end{abstract}

\section{Introduction}

The newly discovered severe acute respiratory syndrome coronavirus 2 (SARS-CoV-2) has triggered the latest outbreak, namely coronavirus disease (COVID-19) (Liu and Zhang 2020). The epidemic disease has affected the social and economical life of the world and has spread rapidly within few months. Several countries witness the serious consequences of the COVID-19 pandemic. Recently, COVID-19 has reiterated in few countries as a second wave with incremental growth. World Health Organization (WHO) has reported that globally 227,940,972 confirmed cases of COVID-19, including 4,682,899 deaths till September 2021 (epidemiological 2021). Thus, COVID-19 detection and diagnosis have received a contemporary research task.

The COVID-19 disease is a type of pneumonia that infects the respiratory system and spreads through close contact. The isolation of infected patients is the preliminary step to break

Jagadeesh Kakarla

jagadeeshk@iiitdm.ac.in

1 Indian Institute of Information Technology, Design and Manufacturing, Kancheepuram, Chennai, India communal spread. In addition to that appropriate medication can increase the survival of patients from death. The detection of COVID-19 infection becomes a tedious task from the physical symptoms like fever, cold, dyspnea, fatigue, and myalgia (Rousan et al. 2020). The reverse transcriptionpolymerase chain reaction (RT-PCR) is a traditional clinical test used for the detection of COVID-19 infection. However, long turnaround time and limited availability of testing kits are the major difficulties with RT-PCR tests (Liu et al. 2020). Thus, the researchers have been motivated for the implementation of automatic COVID-19 detection models. A wide variety of models have been reported for COVID19 detection as follows. Sakib et al. (2020) have generated synthetic chest $\mathrm{x}$-ray images with COVID-19 infection to train the custom $\mathrm{CNN}$ model using generic data augmentation and generative adversarial network. Authors have achieved 93.94\% of accuracy in COVID-19 detection. Horry et al. (2020) have optimized the VGG19 model for COVID-19 detection from x-ray, Ultrasound, and CT scan images. They have attained $86 \%, 100 \%$, and $84 \%$ of precision on $\mathrm{x}$-ray, Ultrasound, and CT scans, respectively. Abbas et al. (2021) have proposed a DeTraC model that investigates class boundaries using a class decomposition mechanism. An accuracy 


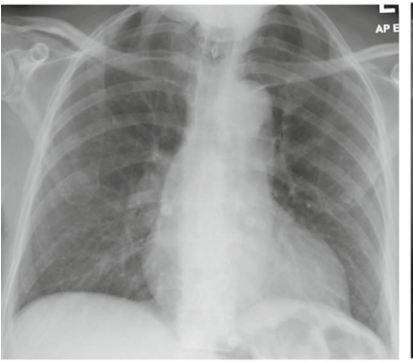

(a) Normal finding

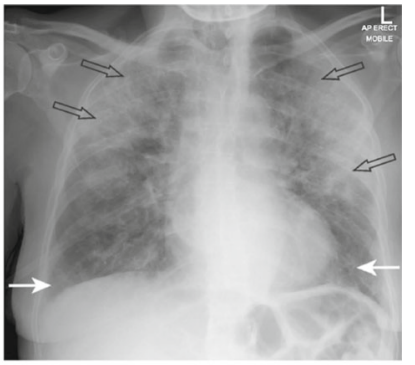

(b) COVID-19 infection

Fig. 1 Chest $\mathrm{x}$-ray for COVID-19 detection

of $93.1 \%$ has been achieved for COVID-19 detection from X-ray images. On the other hand, Erdoğan and Narin (2021) have utilized pre-trained models for feature extraction and the Relieff algorithm for feature selection. They have employed a support vector machine for the classification of cough acoustic signals and achieved $98.4 \%$ accuracy. A transfer learning model with a support vector machine has presented by (Narin 2020) for three-class chest image classification. Similarly, feature selection methods from deep features on $\mathrm{x}$-ray images have devised by (Narin 2021) for COVID-19 detection.

Nowadays, medical imaging plays a vital role in healthcare services for disease detection (Panayides et al. 2020; Hariri and Narin 2021). Some of the health care applications are brain metastases detection (Dikici et al. 2020), ischemic stroke detection (Kodama et al. 2018), ulcer classification (Goyal et al. 2018), and patient risk prediction (Ju et al. 2020). Recent studies have proved that medical imaging technology can be an alternative to RT-PCR as it is highly sensitive for the diagnosis and screening of COVID-19 (Ng et al. 2020) (Liu et al. 2020). Apart from the clinical tests, radiography examination is the fast and cost-effective test for COVID19 detection. Moreover, digital x-ray equipment is available in most hospitals and no need for any additional transportation costs. In general, COVID-19 infection can be identified by the examination of multifocal and bilateral ground-glass opacity and/or consolidation (Rousan et al. 2020; Cleverley et al. 2020). Ground glass opacity is referred to as a region of hazy lung radiopacity in chest radiography. The central mediastinum and heart appear white in a normal chest $\mathrm{x}$ ray and the lungs appear black due to air. There is a change in blackness at the lung portion due to denser ground-glass opacity in COIVD infection.

Figure 1a depicts normal chest $\mathrm{x}$-ray finding while Fig. $1 \mathrm{~b}$ visualizes ground-glass opacity (white arrows) due to COVID-19 infection. Similarly, outlined arrows in Fig. 1b indicates a consolidation of left upper and mid zones of lungs. Thus, the identification of ground-glass opacity and consolidation patterns is essential for COVID-19 detection. Figure 1 also illustrates the effect of COVID-19 on intensity changes near the upper heart region. Thus, the visual examination of these patterns is a challenging task for computer-aided COVID-19 detection systems. Apart from the implementation issues, the class imbalance is one of the significant drawbacks of existing COVID-19 datasets. An insufficient number of COVID-19 positive samples and progressive updates of datasets are major concerns about COVID-19 datasets. The existing proposals have reported their results on balanced datasets having a limited number of COVID19 positive samples. Thus, we have considered two publicly available chest $\mathrm{x}$-ray datasets for COVID-19 detection. The datasets have been chosen such that one dataset consists of data imbalance and the other dataset has balanced data. Details of the two datasets are as follows.

1. COVID-XRay-5K (COVID5K) has created by Minaee et al. (Minaee et al. 2020) and has recently updated with 5184 chest $\mathrm{x}$-ray images. This dataset can be used for binary classification as it contains 5000 normal chest $x$-ray images and 184 COVID-19 positive images. This dataset exhibits data imbalance with a huge number of COVID negative chest $\mathrm{x}$-ray images.

2. Covid radiography (COVIDRD) database of chest $x$-ray images has recently published by Kaggle (Kaggle covid19 2021). This dataset consists of three classes including normal (1341), COVID-19 (1200), and viral pneumonia (1345). As our objective is COVID-19 detection and hence we have considered only normal and COVID-19 positive chest $\mathrm{X}$-ray images. This dataset presents the balanced positive and negative chest $\mathrm{X}$-ray images.

The remaining paper has organized as follows; Sect. 2 presents the literature review. Section 3 elaborates details of the proposed methodology. The quantitative analysis of the proposed model has discussed in Sects. 4 and 5 presents the conclusions of the paper.

\section{Literature review}

The COVID-19 detection from chest $\mathrm{x}$-ray images has become a contemporary research task due to implementation and dataset issues. The deep learning models are popular and successful for image classification. In this section, we have reported the literature review of COVID-19 detection (binary) models. Narin et al. (2021a) have employed pretrained ResNet50 model for the three binary classification tasks including normal/COVID-19, normal/viral pneumonia and normal/bacterial pneumonia. Maghdid et al. (2021) have utilized a modified pre-trained AlexNet model for COVID-19 detection. Jaiswal et al. (2020) have proposed COVIDPEN which is a pruned EfficientNet-based model for COVID-19 detection. Minaee et al. (2020) have presented Deep-COVID using deep transfer learning for prediction of 
Table 1 Review of COVID-19 detection methods

\begin{tabular}{llllll}
\hline References & Year & Method & Sens.\% & Spec.\% & Acc.\% \\
\hline (Narin et al. 2021a) & 2021 & Pre-trained models & - & 98.2 & 96.1 \\
Maghdid et al. (2021) & 2021 & TL and AlexNet & 72 & 100 & 94.1 \\
Jaiswal et al. (2020) & 2020 & TL and DenseNet201 & - & - & 99.82 \\
Minaee et al. (2020) & 2020 & TL and ResNet18 & 98 & 90.7 & - \\
Heidari et al. (2020) & 2020 & TL and VGG16 & 98 & 100 & 94.5 \\
Hemdan et al. (2020) & 2020 & COVIDX-Net & 100 & 80 & - \\
Afshar et al. (2020) & 2020 & COVID-CAPS & - & - & 98.3 \\
\hline
\end{tabular}

COVID-19. Heidari et al. (2020) have performed histogram equalization and bilateral low-pass filter as pre-processing. Then, the classification results have obtained using a transfer learning-based convolutional neural network model. Hemdan et al. (2020) have proposed a COVIDX-Net using modified VGG19 model for COVID-19 detection. Afshar et al. (2020) have implemented a framework known as COVID-CAPS which is based on a capsule network for COVID-19 detection.

Table 1 lists the summary of existing models for binary classification of COVID-19 cases. The implementation of an effective COVID-19 detection system is still a challenging task due to the recent spreading trend of the COVID-19 (Sakib et al. 2020).

Moreover, the implementation of time-efficient models with better performance is another objective for the COVID-19 detection models. It motivated us to implement a time-efficient COVID-19 detection model that can work on two datasets. The major contributions of the work are as follows.

1. Implementation of a time-efficient generalized model for COVID-19 detection that can work on two datasets.

2. The proposed residual separable convolution block improves the performance of the basic MobileNet model.

3. Our proposed model is compatible with the Mobile vision application as it uses MobileNet and produces similar performance with reduced input sizes.

\section{Proposed methodology}

Recently, deep neural networks have been established as successful hands-on models for image classification due to the availability of a huge Imagenet dataset. MobileNet, ResNet, GoogleNet, and Inception ResNet are the most popular models for classification. However, there is a scarcity of large medical image datasets like Imagenet for medical image classification. It motivated researchers to employ transfer learning to improve medical image classification performance. In this section, we have presented the transfer learning procedure along with implementation details of the proposed model.

\subsection{Transfer learning}

Transfer learning involves sharing of weights or knowledge extracted from one problem domain to solve other related problems. High accuracy can be achieved with transfer learning when problems are closely related. The existing pre-trained models are trained with the Imagenet dataset and can perform thousand of class classification. It needs customization of the output layer to handle binary classification. Figure 2a visualizes a pre-trained model for COVID-19 detection and the implementation steps are as follows.

1. Firstly, a pre-trained model is assigned with Imagenet weights and then output layers are removed to customize the output layer.

2. Multi-dimensional feature map obtained from the above pre-trained model is then flattened to generate a onedimensional feature vector.

3. A softmax layer with two neurons is employed to produce the final classification results from the above onedimensional feature vector.

The pre-trained model obtained from the above process needs training with medical image data. Thus, the models are trained with chest $\mathrm{X}$-ray image datasets for the detection of COVID-19 infection.

\subsection{Proposed residual separable convolution block}

In our experiments, we have observed that GoogleNet performs well on both datasets. On the other hand, Inception ResNet exhibits the worst performance on the COVIDRD dataset due to the insufficient size of the dataset. However, MobileNet and ResNet50 produce average performance due to ample convolutions. MobileNet (Howard et al. 2017) is the popular time-efficient model designed especially for mobile vision applications. Our objective is to design a fast COVID-19 detection and hence we have considered pre- 


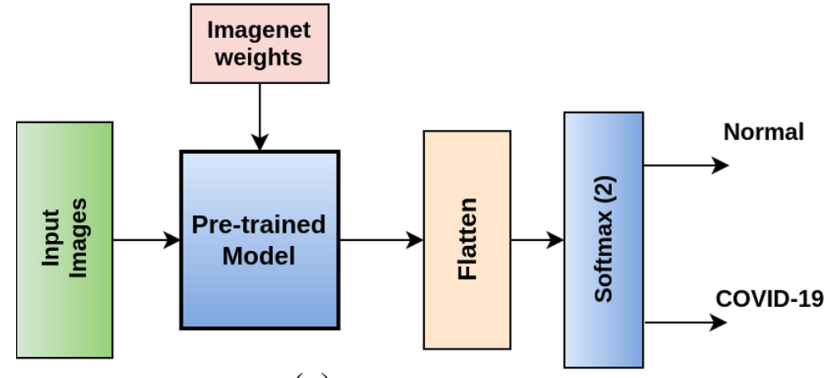

(a) Pre-trained model

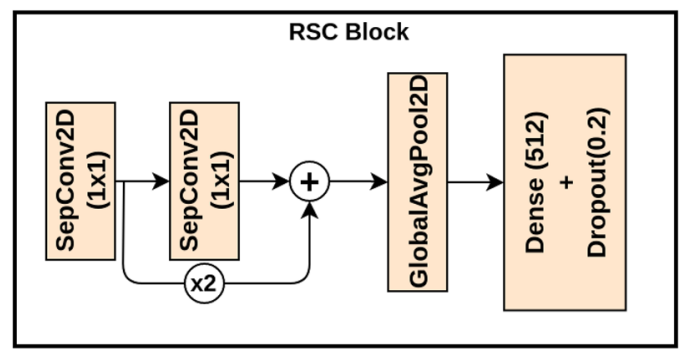

(b) Proposed model

Fig. 2 Block diagram of the proposed model

trained MobileNet. We have proposed a residual separable convolution (RSC) block as shown in Fig. $2 b$ to improve MobileNet performance. In this process, we have replaced flatten layer of the pre-trained MobileNet model with an RSC block. The resulting model is referred to as MobileNet and residual separable convolution block (MNRSC) model. The proposed RSC block uses two separable convolution layers, a global average pooling, a dense layer, and a dropout layer to enhance the spatial feature vectors. We have devised separable convolution with a factored residual connections to reiterate feature maps. Then a global pooling layer converts the multi-dimensional spatial features into a one-dimensional feature vector. In general, the global pooling acts as flatten layer and also avoids over-fitting (Lin et al. 2014). A dense layer with 512 neurons has been employed to establish a traditional neural network. In addition to global average pooling, we have utilized a dropout layer after dense layer to further reduce over-fitting due to class imbalance. The proposed MNRSC model has been designed using MobileNet and an RSC block. The steps involved in the implementation of MNRSC are as follows.

1. Pre-trained MobileNet: It accepts input image $\mathbf{I}(w, h, c)$ of size $(224 \times 224 \times 3)$ and produces feature vector $\mathbf{M N}$ $(x, y, z)$ of size $(7 \times 7 \times 1024)$.

2. RSC block: It transforms $\mathbf{M N}(x, y, z)$ into vector $\mathbf{F}(x)$ as follows.

- Two successive separable convolutions (SC2D) with 512 filters having kernel size of $(1 \times 1)$ are performed to generate feature maps of $\operatorname{SC1}(x, y, z)$ and $\mathbf{S C 2}(x, y, z)$ having dimensions as $(7 \times 7 \times 512)$.

$\operatorname{SC1}(x, y, z)=\operatorname{SC} 2 D(\mathbf{M N}(x, y, z))$

$\operatorname{SC2}(x, y, z)=\operatorname{SC} 2 D(\operatorname{SC1}(x, y, z))$

- Now, factored $\mathbf{S C 1}(x, y, z)$ is added to $\operatorname{SC2}(x, y, z)$ to produce feature vector of size $(7 \times 7 \times 512)$. This step invokes factored residual connection to avoid degradation problem of neurons.

$\mathbf{S C 2}(x, y, z)=\mathbf{S C 2}(x, y, z)+2 * \mathbf{S C 1}(x, y, z)$

- Global average pooling has been employed to generate feature vector $\mathbf{G}(k)$ of size 512. It maps a three-dimensional vector into a one-dimensional vector.

$\mathbf{G}(z)=\frac{\sum_{j=1}^{y} \sum_{i=1}^{x} \mathbf{S C 2}(i, j, z)}{x * y}$

- Finally, a dense layer with 512 neurons forms a fully connected neural network to produce feature vector $\mathbf{F}(z)$ of size 512 .

$\mathbf{F}(z)=\operatorname{Dense}(\mathbf{G}(z))$

3. Classification: Finally, a softmax layer with two neurons has been utilized for binary chest $\mathrm{x}$-ray classification as Normal or COVID-19.

\section{Results and discussion}

In this section, we have presented a detailed analysis of the proposed model using various parameters including input size, and noisy dataset. Four popular fine-tuned models including MobileNet, InceptionResNet, GoogleNet, and ResNet have been considered for the evaluation. The proposed model has been compared with the recent COVID-19 detection model including Minaee et al. (2020) and Maghdid et al. (2021).

\subsection{Experimental setup}

We have evaluated the proposed model using four vital performance metrics like accuracy, sensitivity, specificity, and jaccard similarity. Sensitivity measures the true positive rate while specificity calculates the true negative rate. Accuracy and jaccard similarity focus on overall classification performance. The proposed model and other pre-trained models have implemented using python and tensorflow. All experiments have been conducted on an Intel Xeon processor with a 25 GB GPU system. We have trained the proposed and pretrained models using Adam optimizer with an initial learning rate of 0.0001 . Table 2 lists out the complete hyper-parameter setup utilized for the experiments.

\subsection{Evaluation on COVID5K dataset}

COVID-Xray-5K (COVID5K) dataset is the publicly available dataset published by Minaee et al. Minaee et al. (2020). It 
Table 2 Hyperparameter setup

\begin{tabular}{ll}
\hline Hyperparameter & Value \\
\hline Batch size & 8 \\
Optimizer & Adam \\
Initial learning rate & 0.0001 \\
Number of epochs & 10 \\
\hline
\end{tabular}

Table 3 Fivefold cross-validation on COVID5K dataset

\begin{tabular}{lllll}
\hline Fold & Accuracy & Sensitivity & Specificity & Jaccard \\
\hline 1 & 100.00 & 100.00 & 100.00 & 100.00 \\
2 & 99.86 & 99.86 & 99.85 & 99.72 \\
3 & 99.52 & 99.52 & 99.65 & 99.08 \\
4 & 99.66 & 99.66 & 99.80 & 99.34 \\
5 & 99.61 & 99.61 & 99.60 & 99.27 \\
Avg. & 99.73 & 99.73 & 99.78 & 99.48 \\
Std. & 0.20 & 0.20 & 0.16 & 0.37 \\
\hline
\end{tabular}

consists of 5184 chest $\mathrm{x}$-ray images. The proposed model has evaluated on the dataset and Table 3 lists out cross-validation results. The proposed MNRSC model has achieved 100\% accuracy in the firstfold and $99 \%$ in other folds. Similar performance has observed in other metrics. This table also manifests that the proposed model procures a mean accuracy of $99 \%$ with a standard deviation of $0.2 \%$. Figure 3 depicts training and validation loss of the proposed MNRSC model in the fourthfold. The proposed MNRSC model exhibits a high validation loss in the first threefold and attains fast convergence within ten epochs due to the use of Adam optimizer. Figure 3 demonstrates that the validation loss becomes consistent after the sixthfold. The proposed model achieves consistent loss due to the deployment of a residual separable convolution block.

Figure 4 visualizes the confusion matrix obtained by the proposed model on COVID5K dataset. From this figure, it

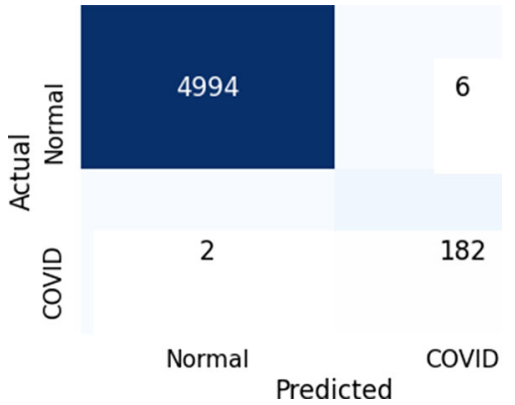

Fig. 4 Confusion matrix on COVID5K dataset

Table 4 Sample x-ray images along with actual and predicted labels

\section{COVID5K}

(1)

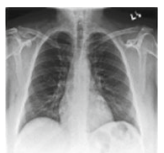

Actual

Predicted
(2)

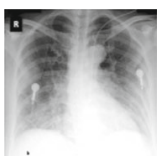

1
(3)

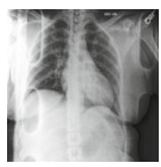

0

can be observed that only two COVID-19 positive samples have been wrongly classified. Whereas, six COVID-19 negative samples are classified wrongly on the COVID5K dataset.

We have investigated the reasons behind the behavior of the proposed model. Table 4 lists out three $\mathrm{x}$-ray image samples from COVID5K dataset. We have found that our proposed model is ineffective with dis-oriented images and can be observed from samples (3) of Table 4. In these samples, other parts like the abdomen have been included due to the wrong orientation. However, our model classifies correctly in case of other images.

Fig. 3 Training and validation loss on COVID5K dataset

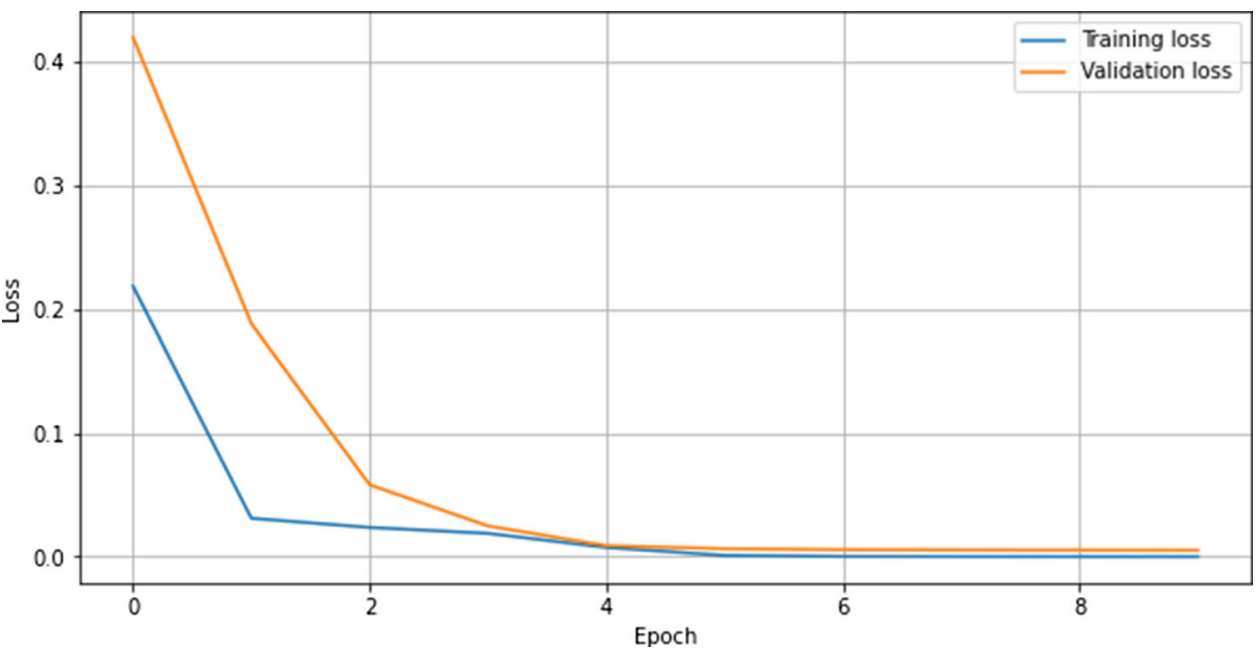


Fig. 5 Performance comparison on COVID5K dataset

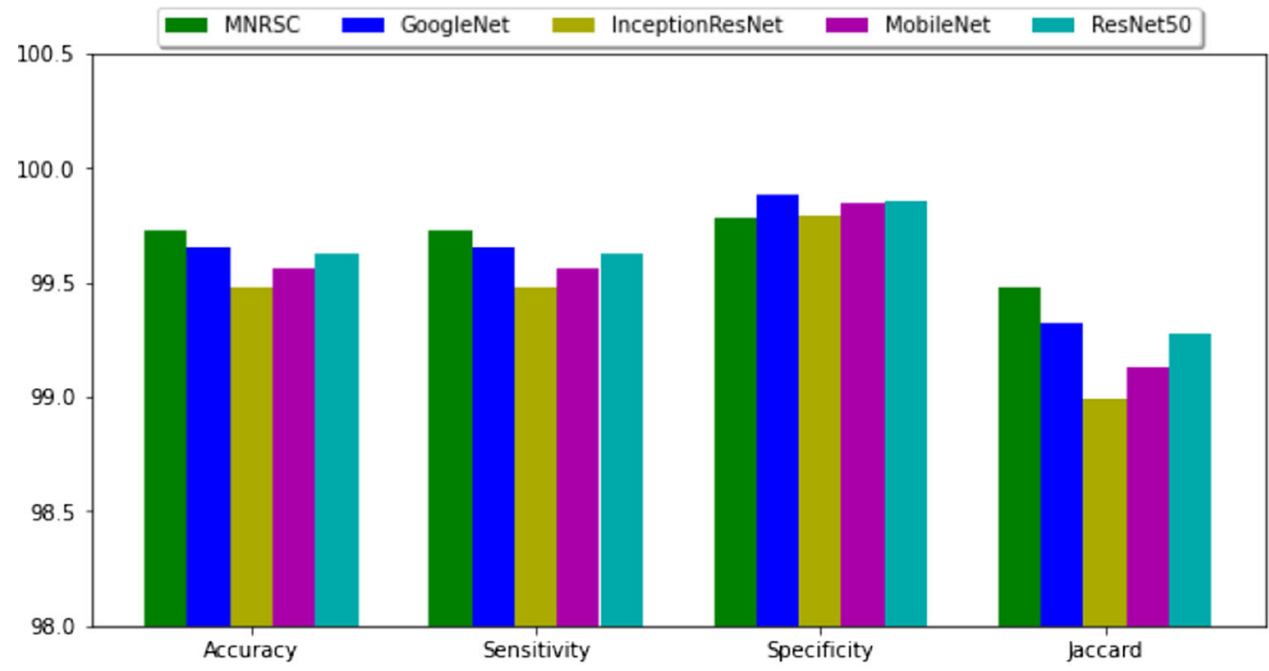

(a) input size $(224 \times 224)$

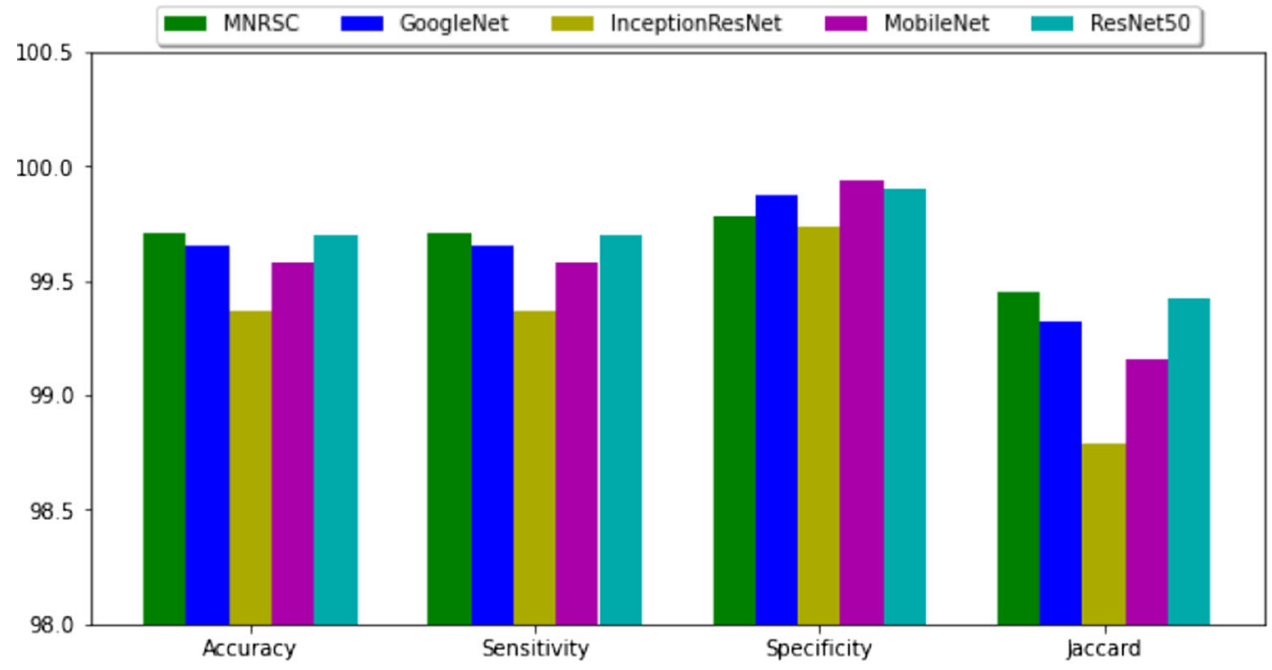

(b) input size $(128 \times 128)$
Table 5 Comparison with state-of-art model

\begin{tabular}{lll}
\hline References & Sensitivity & Specificity \\
\hline Minaee et al. (2020) & 98.00 & 90.70 \\
Maghdid et al. (2021) & 98.77 & 99.02 \\
MNRSC & 99.73 & 99.78 \\
\hline
\end{tabular}

\subsubsection{Comparison with state-of-art model}

The proposed model has been compared with the recent COVID-19 detection models. Authors have reported sensitivity and specificity and hence we also have considered the same metrics for comparison. Table 5 lists out the performance comparison with the state-of-art models. The proposed MNRSC model outperforms the existing model with $99 \%$ of sensitivity and specificity. Our model achieves an improvement of $1 \%$ in sensitivity and $9 \%$ in sensitivity than Minaee et al..

\subsubsection{Comparison with pre-trained models}

We have identified four best-performing pre-trained models including GoogleNet, Inception ResNet, MobileNet, and ResNet50, whose accuracy is greater than $98 \%$. These models have produced similar performance on both datasets after fine-tuning. The input size is the primary factor that influences the computational cost and performance. The low-resolution images are preferred for fast computation especially for time-constrained applications like mobile applications. Thus, we have started our experiments with various input sizes including $(224 \times 224)$ and $(128 \times 128)$ for the 


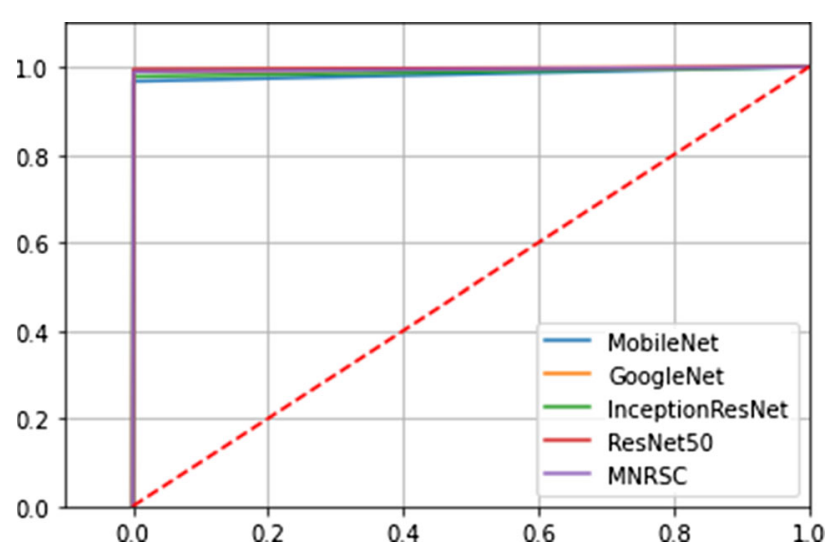

Fig. 6 Comparison of ROC curves

evaluation. The detailed analysis with various input sizes is as follows.

- Performance with input image size $(224 \times 224)$ :

Figure 5a depicts comparison of four metrics with input size $(224 \times 224)$. This figure visualizes that the proposed model outperforms existing pre-trained models in accuracy, sensitivity, and jaccard. The proposed model reports lower specificity than GoogleNet and similar performance on other models.

- Performance with input image size $(128 \times 128)$ :

Figure $5 \mathrm{~b}$ compares the results with input size $(128 \times 128)$ and the proposed model exhibits superior performance on the COVID5K dataset in all metrics except sensitiv- ity. The proposed MNRSC model fails to attain better sensitivity due to data imbalance.

ROC curve visualizes the plot between true positive rate vs false positive rate. Figure 6 visualizes a comparison of ROC curves among the proposed model and its competitive models. The existing pre-trained models report lesser performance on the COVID5K dataset as shown in Fig. 6. However, our model exhibits the best characteristics of its competitive models.

\subsubsection{Performance analysis on noisy data}

In general, noise is another image artifact that influences the performance of the model. Thus, we have evaluated the proposed model on noisy datasets. Medical images suffer from random noise and hence we have imposed the random Gaussian noise on the dataset. We have created a noise image having zero mean with a standard deviation of 5. Table 6 lists out sample noise images along with their original and random noise images. If $I(w, h, c)$ is image, $R N_{\mu, \sigma}(w, h, c)$ is random noise function, then the noise image $N I(w, h, c)$ can be obtained using eq. 1 .

Table 6 Sample noise image generation

(a) Original
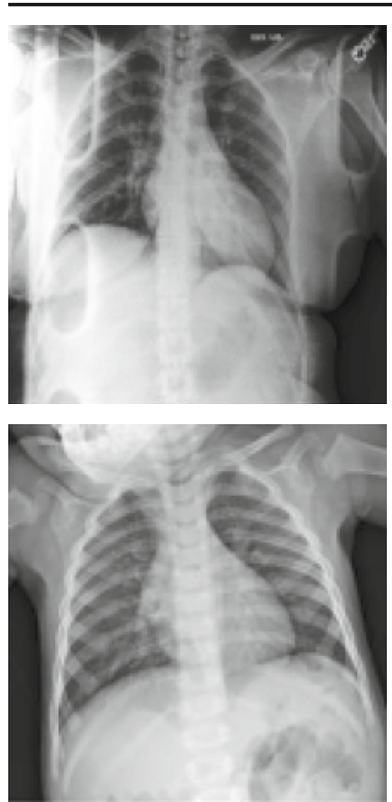

(b) Random noise
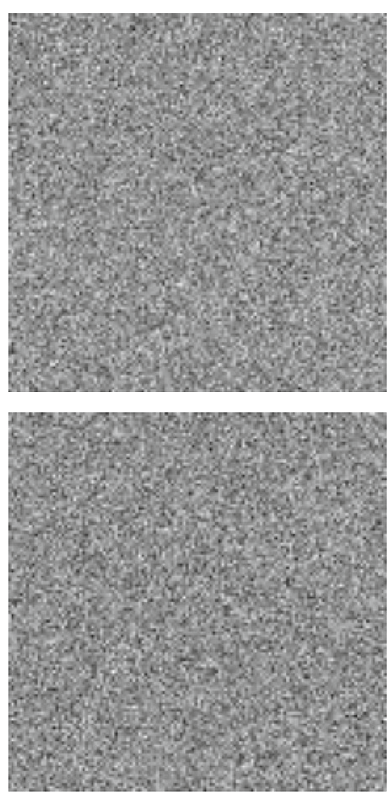

(c) Noise image
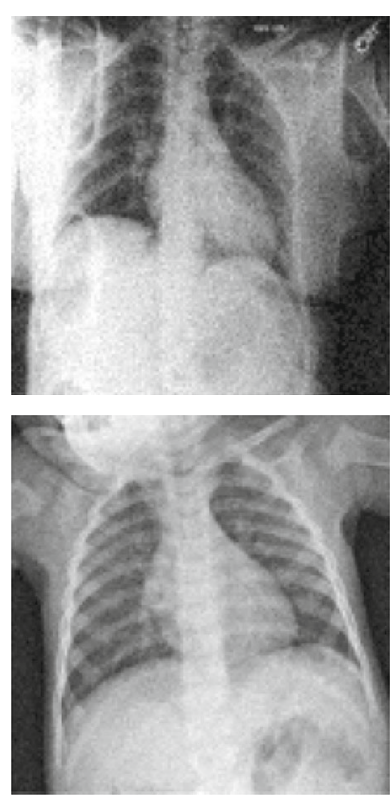
Table 7 Comparison on noisy COVID5K dataset

\begin{tabular}{lllll}
\hline Model & Accuracy & Sensitivity & Specificity & Jaccard \\
\hline GoogleNet & 99.71 & 99.71 & 99.94 & 99.43 \\
InceptionResNet & 99.17 & 99.17 & 99.84 & 98.38 \\
ResNet50 & $\mathbf{9 9 . 8 6}$ & $\mathbf{9 9 . 8 6}$ & 99.88 & $\mathbf{9 9 . 7 3}$ \\
MobileNet & 99.32 & 99.32 & $\mathbf{9 9 . 9 6}$ & 98.66 \\
MNRSC & 99.71 & 99.71 & 99.88 & 99.43 \\
\hline
\end{tabular}

Bold indicates best score

Table 8 Number of parameters and training time

\begin{tabular}{lll}
\hline Model & \# Parameters & Training time (sec.) \\
\hline GoogleNet & $21.905 \mathrm{M}$ & 101.5 \\
InceptionResNet & $54.413 \mathrm{M}$ & 234.2 \\
ResNet50 & $23.788 \mathrm{M}$ & 118.2 \\
MobileNet & $3.329 \mathrm{M}$ & 89.9 \\
MNRSC & $3.626 \mathrm{M}$ & 93.5 \\
\hline
\end{tabular}

$N I(w, h, c)=I(w, h, c)+R N_{\mu=0, \sigma=5}(w, h, c)$

Table 7 compares the results of the proposed model with its competing models on the noisy COVID5K dataset. The proposed MNRSC model has acquired second with $99.71 \%$ accuracy.

\subsubsection{Empirical time complexity}

Training time is another vital factor that needs to be considered while designing effective deep networks. In general, training time mainly depends on the number of parameters and training images. In pre-trained MobileNet, the soft$\max$ layer receives a vector of size $50176(7 \times 7 \times 1024)$ which can be treated as neurons for decision making. A softmax layer maps these 50176 neurons into two neurons for binary classification. In the proposed model, a softmax layer receives only 512 neurons as the RSC block transforms $(7 \times 7 \times 1024)$ vector into 512 neurons. The proposed model uses $0.3 \mathrm{M}$ additional parameters than basic MobileNet. Table 8 evidence that the proposed MNRSC model outperforms its competitive models other than the MobileNet model. However, the proposed MNRSC model takes an additional training time of $4 \mathrm{sec}$. on COIVD5K than MobileNet.

\subsection{Evaluation on COVIDRD dataset}

Covid radiography (COVIDRD) is another dataset published by Kaggle (Kaggle covid-192021). Table 9 lists out the crossvalidation results of the proposed model on the dataset. This table reveals that the proposed model attains $100 \%$ specificity in the first fourfold and $99.81 \%$ in the fifthfold. We have
Table 9 Fivefold cross-validation on COVIDRD dataset

\begin{tabular}{lllll}
\hline Fold & Accuracy & Sensitivity & Specificity & Jaccard \\
\hline 1 & 99.71 & 99.71 & 100.00 & 99.41 \\
2 & 99.80 & 99.80 & 100.00 & 99.61 \\
3 & 99.80 & 99.80 & 100.00 & 99.61 \\
4 & 99.90 & 99.90 & 100.00 & 99.80 \\
5 & 99.61 & 99.61 & 99.81 & 99.22 \\
Avg. & 99.76 & 99.76 & 99.96 & 99.53 \\
Std. & 0.11 & 0.11 & 0.08 & 0.22 \\
\hline
\end{tabular}

achieved a consistent performance of $99 \%$ for other metrics including accuracy, sensitivity, and jaccard.

Figure 7 visualizes the training and validation loss on the COVIDRD dataset. The proposed model reports consistent loss after the sixth epoch and attains optimal loss within ten epochs. The proposed model has classified seven COVID19 positive samples wrongly as negative on the COVIDRD dataset as shown in Fig. 8. However, our model has predicted all COVID-19 negatives samples correctly. Table 10 lists out three $\mathrm{x}$-ray image samples from the COVIDRD dataset. Our model fails to detect dis-oriented images as shown in the sample (3) of Table 10. In this image head of the patient has included due to the wrong orientation.

\subsubsection{Comparison with state-of-art model}

Table 11 lists out the performance comparison with the DeepCOVID model. The Deep-COVID model attains 98.29\%, and $98.02 \%$ of sensitivity, and specificity of respectively. The proposed MNRSC model outperforms the existing model with $99 \%$ of sensitivity and specificity. It proves that our model achieves an improvement of $1 \%$ in both sensitivity and specificity. In medical image classification, even $1 \%$ improvement is considered as a significant performance.

\subsubsection{Comparison with pre-trained models}

Performance comparison with existing pre-trained models is as follows. 

loss on COVIDRD dataset
Fig. 7 Training and validation

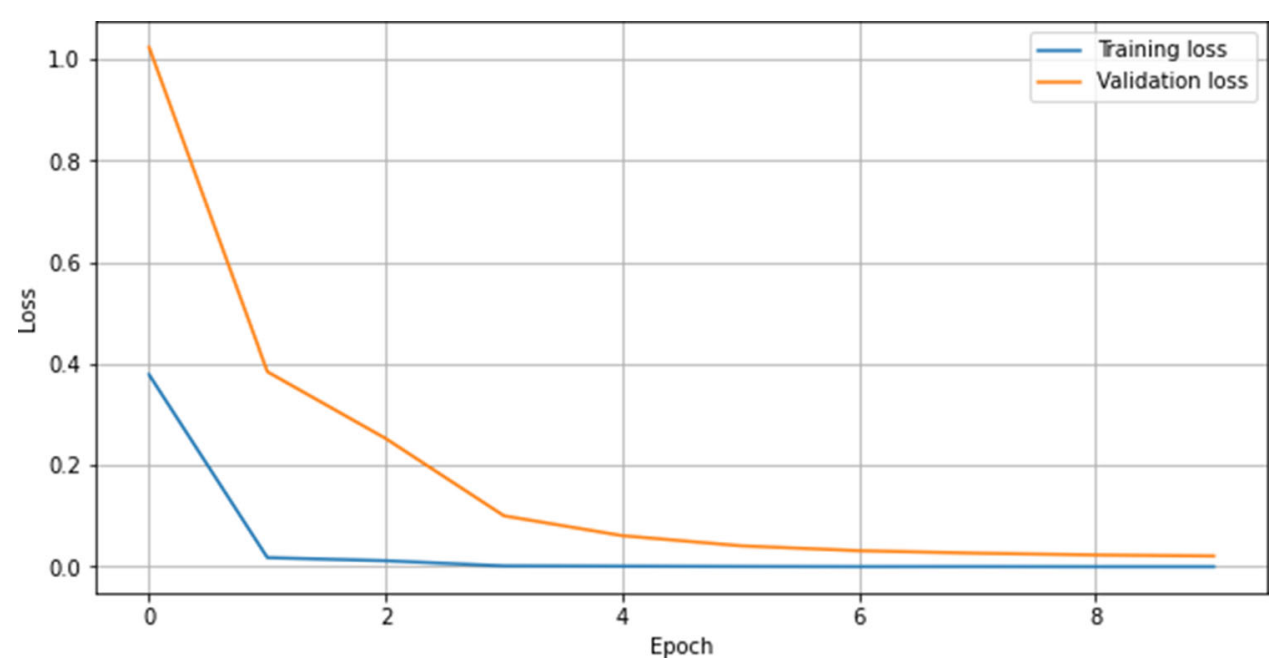

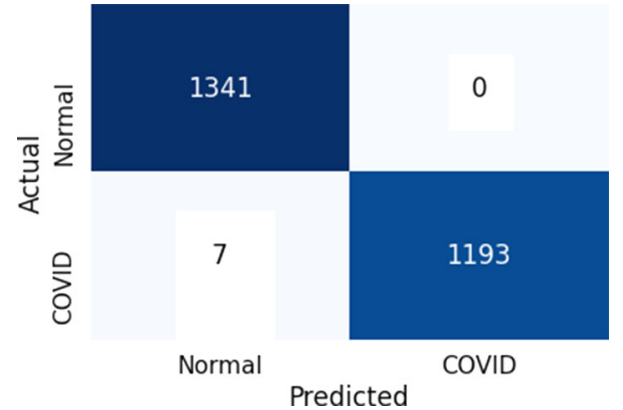

Fig. 8 Confusion matrix on COVIDRD dataset

Table 10 Sample x-ray images along with actual and predicted labels

\section{COVIDRD}

(1)

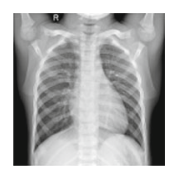

Actual

Predicted

0

0
(2)

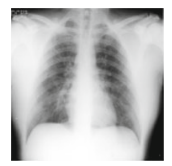

(3)

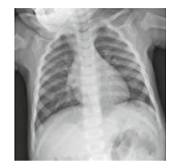

1

0
Table 11 Comparison with state-of-art model

\begin{tabular}{lll}
\hline References & Sensitivity & Specificity \\
\hline Minaee et al. (2020) & 98.29 & 98.02 \\
Maghdid et al. (2021) & 96.16 & 97.49 \\
MNRSC & 99.76 & 99.96 \\
\hline
\end{tabular}

- Performance with input image size $(224 \times 224)$ :

Figure 9a depicts comparison of four metrics with input size $(224 \times 224)$. This figure visualize that the proposed model outperforms existing pre-trained models in all metrics. This figures also highlight that there is a signif-

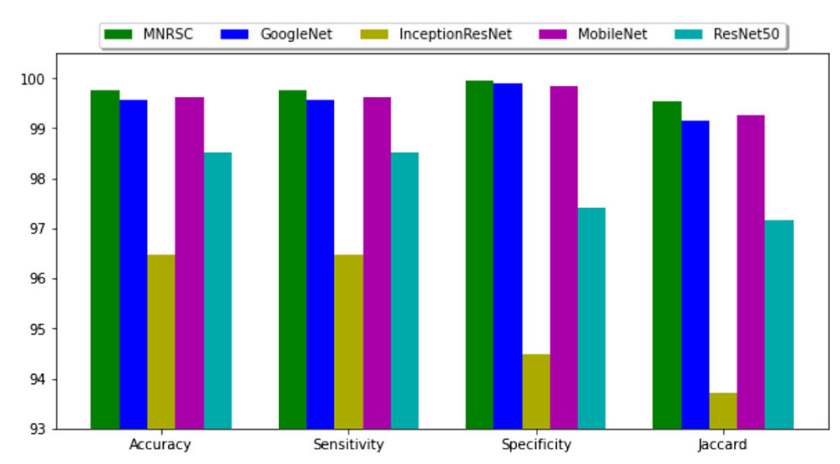

(a) input size $(224 \times 224)$

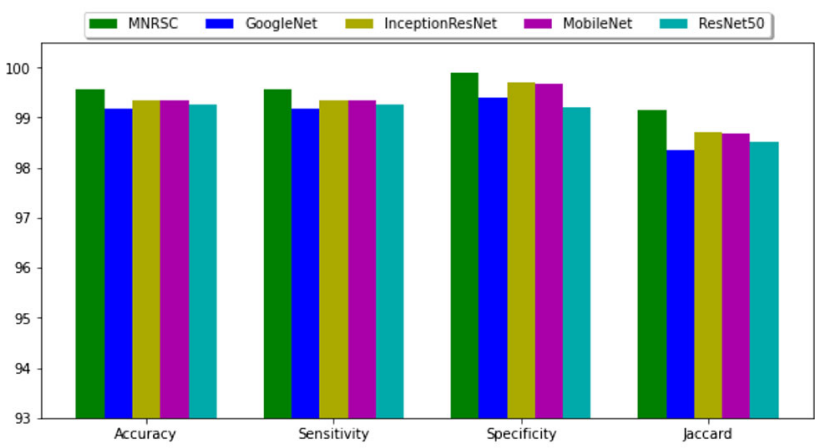

(b) input size $(128 \times 128)$

Fig. 9 Performance comparison on COVIDRD dataset

icant improvement of 7\% than its backbone MobileNet in Jaccard similarity.

- Performance with input image size $(128 \times 128)$ : and the proposed model exhibits superior performance on COVIDRD dataset in all metrics.

Figure 10 visualizes comparison of ROC curves among the proposed model and its competitive models. The proposed
Figure $9 \mathrm{~b}$ compares the results with input size $(128 \times 128)$ 


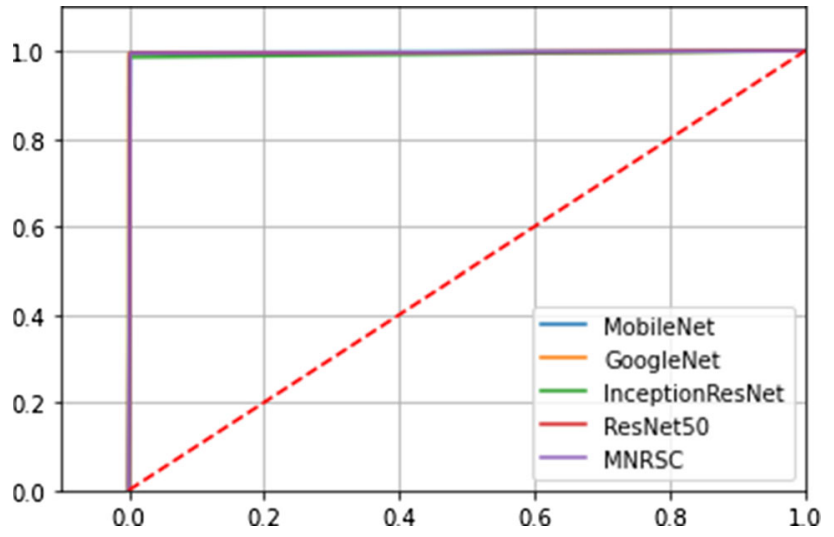

Fig. 10 Comparison of ROC curves

Table 12 Comparison on noisy COVIDRD datasets

\begin{tabular}{lllll}
\hline Model & Accuracy & Sensitivity & Specificity & Jaccard \\
\hline GoogleNet & 99.53 & 99.53 & 99.70 & 99.06 \\
InceptionResNet & 99.61 & 99.61 & 99.70 & 99.22 \\
ResNet50 & 99.53 & 99.53 & 99.78 & 99.06 \\
MobileNet & 99.25 & 99.25 & 98.96 & 98.52 \\
MNRSC & $\mathbf{9 9 . 6 5}$ & $\mathbf{9 9 . 6 5}$ & $\mathbf{1 0 0 . 0}$ & $\mathbf{9 9 . 2 9}$ \\
\hline
\end{tabular}

Bold indicates best score

Table 13 Number of parameters and training time

\begin{tabular}{lll}
\hline Model & \# Parameters & Training time (sec.) \\
\hline GoogleNet & $21.905 \mathrm{M}$ & 51.0 \\
InceptionResNet & $54.413 \mathrm{M}$ & 116.5 \\
ResNet50 & $23.788 \mathrm{M}$ & 58.9 \\
MobileNet & $3.329 \mathrm{M}$ & 44.5 \\
MNRSC & $3.626 \mathrm{M}$ & 46.7 \\
\hline
\end{tabular}

model exhibits similar performance as pre-trained models on the COVIDRD dataset as shown in Fig. 10.

\subsubsection{Performance analysis on noisy datasets}

Our model outperforms its competing models on a noisy COVIDRD dataset with $99.65 \%$ accuracy and can be observed from Table 12.

\subsubsection{Empirical time complexity}

Table 13 shows that the proposed MNRSC model outperforms its competitive models other than the MobileNet model. However, the proposed MNRSC model takes an additional training time of $2 \mathrm{sec}$. on COIVDRD dataset than MobileNet.

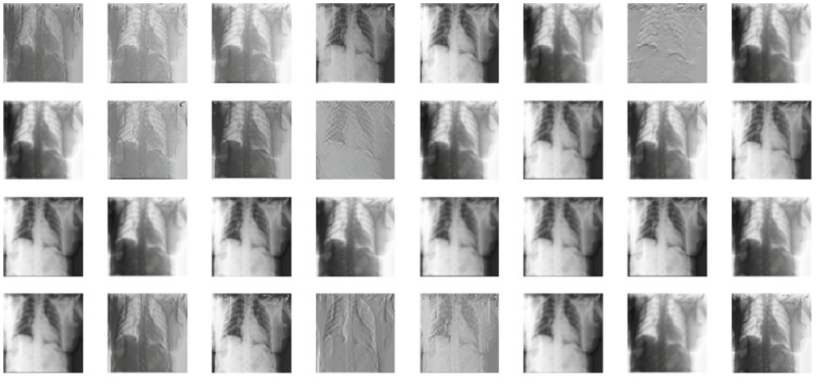

(a) Normal
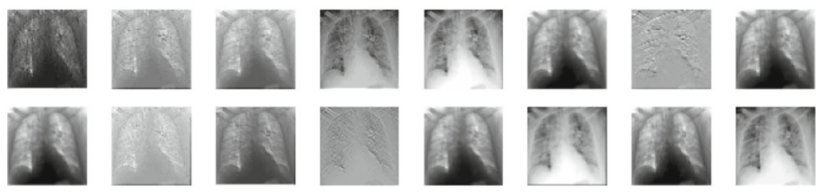

(2)
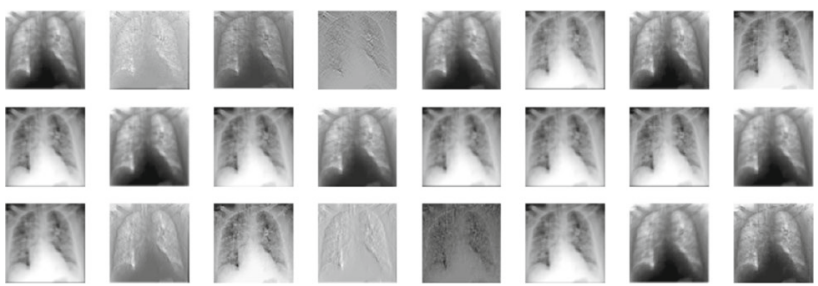

(b) COVID-19

Fig. 11 Convolution results at first layer

\subsection{Discussion}

We have analyzed high-level feature maps of the proposed model. Figure 11a and $\mathrm{b}$ depict convolution results after the first layer on Normal and COVID-19 image samples, respectively. These figures visualize the structural, edge, and contrast features of the images. Consider the feature map in the first-row seventh column of Fig. 11. Where the structure of the skeleton and heart has degenerated. Thus, these samples have resulted in the wrong classification. The proposed model exhibits better sensitivity on COVID5K and better specificity on the COVIDRD dataset. However, the difference between sensitivity and specificity is negligible and hence our model exhibits optimal performance. Moreover, our model produces the best results on a balanced dataset like COVIDRD. On the other hand, it achieves competitive performance on imbalanced datasets like COVID5K. The proposed residual separable convolution block over MobileNet achieves significant performance on both datasets. Further, the following conclusions can be made from the above analysis.

- Our model produces better results on a balanced dataset and competitive results on an imbalanced dataset.

- Our model exhibits superior performance in accuracy, sensitivity, and Jaccard on the COVID5K dataset.

- Our model outperforms existing models in all key metrics on the COVIDRD dataset. 
- It also reports consistent results with various input sizes and hence it is compatible with low-scale devices like mobiles.

- The proposed model attains a 7\% improvement in jaccard similarity than the MobileNet model on the COVIDRD dataset.

\section{Conclusion}

COVID-19 detection from x-ray images has become contemporary research due to an increase in the number of COVID-19 cases and imbalanced datasets. However, the implementation of time-efficient models with better performance is still challenging. In this work, we have proposed a MobileNet and residual separable convolution block model for chest $\mathrm{x}$-ray image classification. The proposed residual separable convolution block uses two separable convolutions, global average pooling, a dense layer, and a dropout layer. The separable convolutions with a factored residual connection have been utilized to take advantage of computational cost and feature enhancement. Global average pooling has been devised instead of Flatten layer to consider image-level features. Two publicly available datasets have been considered for the performance evaluation of the proposed model. The proposed model outperforms existing pre-trained models and state-of-art models with $99 \%$ accuracy in all key metrics except specificity. However, the difference between sensitivity and specificity is negligible and hence our model exhibits optimal performance. The proposed model achieves similar results on noisy datasets. Our proposed model incurs less training time than the existing pre-trained model and exhibits competitive performance as basic MobileNet. Further, the proposed model is compatible with mobile applications as it uses fewer parameters and lesser training time.

The proposed model fails to exhibit superior performance on the noisy dataset with imbalanced data. In our future work, we have planned to design an efficient COVID-19 detection model for low quality and noisy datasets.

\section{Declarations}

Conflicts of interest The authors declare that there is no conflict of interest regarding the publication of this paper.

Ethical approval This article does not contain any studies with human participants or animals performed by any of the authors.

Informed consent Informed consent was obtained from all individual participants included in the study.

\section{References}

Abbas A, Abdelsamea MM, Gaber MM (2021) Classification of COVID-19 in chest $\mathrm{x}$-ray images using DeTraC deep convolutional neural network. Appl Intell 51(2):854

Afshar P, Heidarian S, Naderkhani F, Oikonomou A, Plataniotis KN, Mohammadi A (2020) Covid-caps: a capsule network-based framework for identification of covid-19 cases from x-ray images. Pattern Recognit Lett 138:638

Cleverley J, Piper J, Jones MM (2020) The role of chest radiography in confirming covid-19 pneumonia. BMJ, vol 370

Dikici E, Ryu JL, Demirer M, Bigelow M, White RD, Slone W, Erdal BS, Prevedello LM (2020) Automated brain metastases detection framework for T1-weighted contrast-enhanced 3D MRI. IEEE J Biomed Health Inform 24(10):2883

Erdoğan Y.E, Narin A (2021) COVID-19 detection with traditional and deep features on cough acoustic signals. Comput Biol Med, $p$ 104765

Goyal M, Reeves ND, Davison AK, Rajbhandari S, Spragg J, Yap MH (2018) Dfunet: convolutional neural networks for diabetic foot ulcer classification. IEEE Trans Emerg Topics Comput Intell 4(5):728

Hariri W, Narin A (2021) Deep neural networks for COVID-19 detection and diagnosis using images and acoustic-based techniques: a recent review. Soft Comput, pp 1-18

Heidari M, Mirniaharikandehei S, Khuzani AZ, Danala G, Qiu Y, Zheng B (2020) Improving the performance of CNN to predict the likelihood of COVID-19 using chest $\mathrm{x}$-ray images with preprocessing algorithms. Int J Med Inform 144:104284

Hemdan EED, Shouman MA, Karar ME (2020) arXiv preprint arXiv:2003.11055

Horry MJ, Chakraborty S, Paul M, Ulhaq A, Pradhan B, Saha M, Shukla N (2020) COVID-19 detection through transfer learning using multimodal imaging data. IEEE Access 8:149808. https:// doi.org/10.1109/ACCESS.2020.3016780

Howard A.G, Zhu M, Chen B, Kalenichenko D, Wang W, Weyand T, Andreetto M, Adam H (2017) arXiv preprint arXiv:1704.04861

Jaiswal AK, Tiwari P, Rathi VK, Qian J, Pandey HM, Albuquerque VHC (2020) Medrxiv

Ju R, Zhou P,Wen S, Wei W, Xue Y, Huang X, Yang X (2020) 3D-CNNSPP: A Patient Risk Prediction System From Electronic Health Records via 3D CNN and Spatial Pyramid Pooling. IEEE Trans Emerg Topics Comput Intell

Kaggle covid-19 radiography database. https://www.kaggle.com/ tawsifurrahman/covid19-radiography-database. Accessed: 202101-07

Kodama T, Kamata K, Fujiwara K, Kano M, Yamakawa T, Yuki I, Murayama Y (2018) Ischemic stroke detection by analyzing heart rate variability in rat middle cerebral artery occlusion model. IEEE Trans Neural Syst Rehabilitat Eng 26(6):1152

Lin M, Chen Q, Yan S (2014) An occlusion probability model for improving the rendering quality of views. Netw Netw

Liu H, Liu F, Li J, Zhang T, Wang D, Lan W (2020) Clinical and CT imaging features of the COVID19 pneumonia: focus on pregnant women and children. J Infect 80(5):e7

Liu X, Zhang S (2020) COVID19: Face masks and human-to-human transmission. Influenza Other Respirat Virus

Maghdid HS, Asaad AT, Ghafoor KZ, Sadiq AS, Mirjalili S, Khan MK (2021) Multimodal image exploitation and learning 2021, vol 11734 (International Society for Optics and Photonics, 2021), vol 11734, p 117340E

Minaee S, Kafieh R, Sonka M, Yazdani S, Soufi GJ (2020) Deep-covid: Predicting covid-19 from chest X-ray images using deep transfer learning. Med Image Anal 65:101794 
Narin A (2020) Medical technologies congress (TIPTEKNO) (IEEE, 2020), pp 1-4

Narin A (2021) Automatic detection of coronavirus disease (covid19) using X-ray images and deep convolutional neural networks. Comput Biol Med, p 104771

Narin A, Kaya C, Pamuk Z (2021) Automatic detection of coronavirus disease (covid-19) using X-ray images and deep convolutional neural networks. Pattern Anal Appl, pp 1-14

Ng MY, Lee EY, Yang J, Yang F, Li X, Wang H, Lui MM, Lo CSY, Leung B, Khong PL et al (2020) Radiol Cardiothoracic Imag 2(1): e200034

Panayides AS, Amini A, Filipovic ND, Sharma A, Tsaftaris SA, Young A, Foran D, Do N, Golemati S, Kurc T et al (2020) IEEE J Biomed Health Inf 24(7):1837
Rousan LA, Elobeid E, Karrar M, Khader Y (2020) Chest X-ray findings and temporal lung changes in patients with COVID-19 pneumonia. BMC Pulmonary Med 20(1): 1

Sakib S, Tazrin T, Fouda MM, Fadlullah ZM, Guizani M (2020) DL-CRC: deep learning-based chest radiograph classification for COVID-19 detection: a novel approach. IEEE Access 8:171575

Weekly epidemiological update on covid-19 - 30 march 2021. https:// www.who.int/publications/m/item/weekly-operational-updateon-covid-19---20-september-2021. Accessed: September 2021

Publisher's Note Springer Nature remains neutral with regard to jurisdictional claims in published maps and institutional affiliations. 